\title{
Shear Bond Strength of Orthodontic Brackets Cured with Different Light Sources under Thermocycling
}

Oral Sokucua

Seyda Herguner Siso ${ }^{b}$

Firat Ozturkc

Ruhi Nalcaci ${ }^{d}$

\section{ABSTRACT}

Objectives: The aim of this study was to compare the shear bond strength (SBS) of two different light sources under different thermal cycle frequencies.

Methods: Ninety human premolar teeth were divided into six groups, in which three groups consisted of halogen groups - Group I was cured with halogen without thermocycle, Group II cured with halogen with 500 thermocycles, and Group III cured with halogen with 10,000 thermocycles. The other three groups consisted of Plasma arc (PAC) group - Group IV was cured with PAC without thermocycle, Group V cured with PAC thermocycled 500 cycles, and Group VI cured with PAC thermocycled 10,000 cycles. Two way ANOVA was used to compare the SBS between the groups, and chi squaretest was used to compare the bond failure of ARI scores among the groups.

Results: A significant difference was found for curing lights $(P<.001)$ and thermocycling $(P<.01)$. However, there was no interaction between light curing and thermocycling $(P=.177)$. The halogen groups demonstrated higher mean SBS than the PAC groups. Both groups showed a significant reduction between no cycles and 10,000 cycles $(P<.05)$.

Conclusions: Both light sources showed favorable SBS performance without the thermocycle procedure. Thermocycling significantly decreased the SBS values of both groups. (Eur J Dent 2010;4:257262)

Key words: Shear bond strength; ARI score; Thermocycling; Halogen; Plasma arc.

assistant Professor, Department of Orthodontics, University of Gaziantep, Gaziantep, Turkey.

b Assistant Professor, Department of Restorative Dentistry, University of Cumhuriyet, Sivas, Turkey. Assistant Professor, Department of Orthodontics, University of Inonu, Malatya, Turkey.

d Assistant Professor, Department of Orthodontics, University of Suleyman Demirel, Isparta, Turkey.

- Corresponding author: Dr. Oral Sokucu Gaziantep Universitesi, Dishekimligi Fakultesi, Ortodonti AD, 27310, Gaziantep, Turkey. Phone: +903423606060 E-mail: almanoraldahotmail.com

\section{INTRODUCTION}

With the increasing use of straight wire or preadjusted appliances in orthodontics, practitioners are switching their focus from wire bending to bracket positioning. In fixed-appliance treatment, one of the most important requirements is correct bracket positioning. ${ }^{1}$ Bonding orthodontic brackets with visible light-cured adhesives was first reported by Tavas and Watts. ${ }^{2}$ The advantage of a light-cured adhesive system is that it gives the clinician the ideal working time to position the brack- 
et, reduces the risk of contamination, and helps in easy removal of excess material after bonding. ${ }^{3}$

Most sources of visible blue light applied in dentistry use tungsten-filament halogen lamps that incorporate a blue filter to produce light of 400-500 nm. The basic principle of light conversion by the halogen technique is inherently inefficient. ${ }^{4}$ The disadvantages of conventional halogen units are the degradation of the lamp, the filter, and the reflector, leading to reduced curing effectiveness. ${ }^{5}$ They have a limited lifetime of 100 hours. Filters can undergo blistering, and reflectors can discolor. The prolonged curing time with halogen bulbs can be uncomfortable to the patient, impractical with children, and inconvenient for the clinician. ${ }^{5,6}$

In the 1990s, rapid light-curing alternatives to the conventional halogen units, such as quartztungsten halogen (QTH), plasma arc curing light (PAC), and light-emitting diode (LED) were introduced in orthodontics. ${ }^{7}$

The reduced curing achieved when the PAC light is used to bond the orthodontic bracket is advantageous for both the patient and the clinician. ${ }^{3}$ Manufacturers have declared that the PAC lamp in restorative dentistry reduces the time needed to cure the composite resin filling materials from 30-40 seconds to as little as 1-3 seconds. However, conventional halogen lamps emit white light, which is filtered to produce blue light with a wavelength of 400-500 $\mathrm{nm}$ at an energy level of approximately $300 \mathrm{~mW}$. The PAC lamp has a much higher peak energy level of $900 \mathrm{~mW}$ with a narrower spectrum of $430-490 \mathrm{~nm} .^{8}$

Studies performed under ideal laboratory conditions do not describe how the materials might act in the oral cavity. Clinically, intraoral contamination, moisture, and temperature can influence the bond strength. Therefore, thermocycling is used to simulate clinical conditions and enhance the human applicability of bonding agents. ${ }^{9}$ Previous studies have evaluated the effect of thermal cycling. ${ }^{10,11}$ However, the various numbers of cycles under different light sources has not been evaluated in detail.

The aim of this study was to compare the shear bond strength (SBS) of two different light sources under different thermal cycle frequencies.

\section{MATERIALS AND METHODS}

Two different light units for curing an orthodontic bracket adhesive were compared: a PAC light-curing system (Apollo 95E Dental Medical Diagnostics, Woodland Hills, California, USA) and a conventional halogen light-curing system (Hilux dental, Ankara, Turkeyl. Apollo 95E is a PAC that emits light in a narrow band between $440 \mathrm{~nm}$ and $495 \mathrm{~nm}$, according to the manufacturer's instructions for use. With the standard light guide $18 \mathrm{~mm}$ in diameter), an intensity of $980 \mathrm{~mW} \mathrm{~cm}^{2}$ was measured with an Optilux radiometer when the light guide was placed directly on the detector. The Hilux dental is a halogen light cure with a wavelength between $400 \mathrm{~nm}$ and $515 \mathrm{~nm}$. The standard light guide ( $8 \mathrm{~mm}$ in diameter) with an intensity of $500 \mathrm{~mW} \mathrm{~cm}^{2}$ was measured through checking the Optilux radiometer.

Ninety human maxillary premolar teeth extracted for orthodontic reasons were cleaned of debris and stored in distilled water. The criteria for tooth selection were intact buccal enamel; no pretreatment of chemical agents, such as derivatives of peroxide, acid, or alcohol; no cracks from forceps; no caries; and no restorations. The teeth were stored in distilled water continuously after extraction. The water was changed weekly to avoid bacterial growth. Before bonding, the labial surfaces of the teeth in all groups were polished using non-fluoride pumice, rinsed with water, and dried with an air spray. The teeth were embedded in phenolic rings using autopolymerizing polymethyl methacrylate. A mounting jig was used to align the facial surface of the tooth to be perpendicular to the bottom of the mold and its labial surface parallel to the force during the SBS test.

Before bonding, the teeth were randomly divided into six groups, each containing 15 teeth. The bonding surface of each tooth was pumiced for 10 seconds and rinsed for 10 seconds with distilled water. Fifteen teeth were etched for 20 seconds with $37 \%$ phosphoric acid, washed with a spray for 10 seconds, and dried to a chalky-white appearance, and subsequently, the sealant was applied to the etched surface. The surface was thoroughly dried, and a thin layer of orthodontic adhesive primer (Transbond XT'TM, 3M Unitek, Monrovia, California, USA) was applied. An orthodontic composite resin (Transbond $\mathrm{XT}^{\mathrm{TM}}$ adhesive paste) was used for all teeth. 
In this study, orthodontic premolar metal brackets (Generus Roth, GAC, NY, USA) were used. The average bracket surface area of the bracket base was determined to be $12.4 \mathrm{~mm}^{2}$. Each bracket was placed on the tooth with a constant force by one operator (O.S.).

Group I: Brackets were cured with the halogen light for 20 seconds and stored in distilled water for 24 hours.

Group II: Brackets were cured with the halogen light for 20 seconds and thermocycled in water between $5 \pm 2^{\circ} \mathrm{C}$ and $50 \pm 2^{\circ} \mathrm{C}$ for 500 cycles.

Group III: Brackets were cured with the halogen light for 20 seconds and thermocycled in water between $5 \pm 2^{\circ} \mathrm{C}$ and $50 \pm 2^{\circ} \mathrm{C}$ for 10,000 cycles.

Group IV: Brackets were cured with the PAC light for 6 seconds ( $3 \mathrm{~s}$ mesial and $3 \mathrm{~s}$ distal) and stored in distilled water for 24 hours.

Group V: Brackets were cured with the PAC light for 6 seconds ( $3 \mathrm{~s}$ mesial and $3 \mathrm{~s}$ distal) and thermocycled in water between $5 \pm 2^{\circ} \mathrm{C}$ and $50 \pm 2^{\circ} \mathrm{C}$ for 500 cycles.

Group VI: Brackets were cured with the PAC light for 6 seconds ( $3 \mathrm{~s}$ mesial and $3 \mathrm{~s}$ distal) and thermocycled in water between $5 \pm 2^{\circ} \mathrm{C}$ and $50 \pm 2^{\circ} \mathrm{C}$ for 10,000 cycles.

The exposure to each bath was 30 seconds and the transfer time between the two baths was $5-10$ seconds. 500 cycles between $5^{\circ} \mathrm{C}$ and $50^{\circ} \mathrm{C}$ were in accordance with the recommendation of the International Organization for Standardization (ISO/TS 11405). ${ }^{12}$ The other 10,000 cycles were performed to demonstrate long-term exposure to moisture at oral temperature.

The PAC light was calibrated by inserting the curing tip completely into the calibration port and then depressing the hand switch. The halogen light was calibrated by placing the fiber-optic probe directly on the top of the built-in sensor until the light indicated that the probe intensity was adequate.

A universal testing machine (LF Plus, LLOYD Instruments, Ametek Inc., England) was used for the shear bond test at a crosshead speed of $1 \mathrm{~mm} /$ min. Force was applied directly to the brackettooth interface using the flattened end of a steel rod. The load at failure was recorded by a personal computer connected to the test machine. SBS values were calculated as the recorded failure load divided by the surface area (bracket base) and were expressed in megapascals (MPa).

After debonding, the enamel surface of each tooth and the bracket bases were examined with a stereomicroscope (magnification $\times 10$ ) by one investigator (S.H.S.) to determine the amount of residual adhesive remaining on each tooth. The adhesive remnant index (ARI) was used to assess the amount of adhesive left on the enamel surfaces. ${ }^{10}$ This scale ranges from 0 to 3 . A score of 0 indicates no adhesive remaining on the tooth in the bonding area, 1 indicates less than half of the adhesive remaining on the tooth, 2 indicates more than half of the adhesive remaining on the tooth, and 3 indicates all adhesive remaining on the tooth with a distinct impression of the bracket mesh.

\section{Statistical analysis}

Two-way analysis of variance was used to obtain the significant differences among curing lights, thermocycling, and their interactions. All treatment combination means for bond strength values were compared using the Tukey multiple comparison test $(\alpha=.05)$. The chi-square test was used to compare the bond failure of ARI scores among the groups.

\section{RESULTS}

The two-way analysis of variance showed a significant difference for curing lights $(P<.001)$ and thermocycling $(P<.01)$. However, there was no interaction between light curing and thermocycling ( $P=$.177). The statistical results of SBS are presented in Tables I and II. It was found that the groups that did not undergo the thermocycle process (Groups I and IV) revealed higher SBS values than the thermocycled groups.

The comparison of both the groups indicated that the halogen groups demonstrated higher mean SBS than the PAC groups. Both groups showed a significant reduction between no cycles and 10,000 cycles $(P<.05)$.

Table III shows the distribution of ARI scores expressed as the frequency of occurrence. The halogen groups (I, II, III) and plasma groups (IV, V, VI) predominantly had ARI scores of 1 . This result indicated that the brackets failed at the enameladhesive interface. It is shown that after 10,000 cycles, the ARI scores shifted to 1 in the PAC group. Contrary to this, the halogen group shifted to score 0 in the same cycle. The chi square-test 
demonstrated no statistical difference between both the groups ( $P>$.05).

\section{DISCUSSION}

The purpose of this study was to compare the SBS with halogen and PAC after being thermocycled. Previous studies showed that thermocycling and water storage affected the SBS of brackets. ${ }^{10,11}$ In the absence of these procedures, the SBS testing provides only the initial bond strength data. Therefore, it is important to include thermal cycling of the specimens to assess the durability of the bond.

There was no statistical difference in the SBS values between the two light sources without thermocycled procedure. Reynolds ${ }^{13}$ indicated that the minimum SBS for the orthodontic treatment is from 5.9 to $7.9 \mathrm{MPa}$. Furthermore, our results showed that without thermocycling, both light sources have acceptable SBS values (Halo- gen light: 21.20 MPa, PAC: 19.72 MPa). Oesterle et $\mathrm{al}^{14}$ reported that $\mathrm{PAC}$ is four times more intense than halogen light. The PAC exposure time of 6-9 $\mathrm{s}$ produces SBS equal to those produced in $40 \mathrm{~s}$ by a conventional halogen curing light in vitro. In another study, Pettemerides et $\mathrm{l}^{8}$ compared the failure rates in both PAC and halogen lights in an in-vitro study and observed no differences. It was shown that the PAC had acceptable SBS values compared to those for the halogen light with the advantage of reducing chair time.

After 500 thermocycles, a decrease in SBS was observed in both groups. The decrease in SBS was around 6 percent in the halogen group and 28 percent in the PAC group. Daub et $\mathrm{al}^{15}$ observed a 16.7 percent decrease after 500 thermocycles with the halogen-light procedure. However, Arıcı and $\mathrm{ArICl}^{16}$ found only a 5.7 percent reduction in the bond strength after 200 thermocycles.

The theories of the reduction after thermocy-

Table 1. Two-way analysis of variance of force (MPa) required in debonding metal brackets

\begin{tabular}{|c|c|c|c|c|c|}
\hline & Type III SS & $d f$ & MS & $\mathrm{F}$ & Sig. \\
\hline Type of curing & 507.38 & 2 & 253.6 & 7.6 & .001 \\
\hline Thermocycling & 256.7 & 1 & 256.7 & 7.7 & .007 \\
\hline $\begin{array}{l}\text { Curing light* } \\
\text { thermocycling }\end{array}$ & 116.6 & 2 & 58.3 & 1.7 & .290 \\
\hline
\end{tabular}

Table 2. Mean and standard deviations (SD) for each group.

\begin{tabular}{lccc}
\hline & \multicolumn{1}{c}{ Thermocycling status } \\
\hline & $\begin{array}{c}\text { No cycles } \\
\text { Mean (SD) }\end{array}$ & $\begin{array}{c}500 \text { cycles } \\
\text { Mean (SD) }\end{array}$ & $\begin{array}{c}10,000 \text { cycles } \\
\text { Mean (SD) }\end{array}$ \\
\hline Halogen group & $21.2( \pm 5.1) \mathrm{A}$ & $19.72( \pm 5.7) \mathrm{B}$ & $14.92( \pm 5.8) \mathrm{C}$ \\
Plasma group & $18.88( \pm 6.3) \mathrm{B}$ & $13.64( \pm 6.9) \mathrm{C}$ & $13.18( \pm 3.8) \mathrm{C}$ \\
\hline
\end{tabular}

* Groups shown with different letters were significant at $P=.05$, according to Tukey's HSD test.

Table 3. Frequency and percentage occurrence of the adhesive remnant index (ARI) for each group. A score of 0 indicates no adhesive remaining on the tooth in the bonding area, 1 indicates less than half of the adhesive remaining on the tooth, 2 indicates more than half of the adhesive remaining on the tooth, and 3 indicates all adhesive remaining on the tooth with a distinct impression of the bracket mesh.

\begin{tabular}{|c|c|c|c|c|c|}
\hline Groups & $\mathrm{N}$ & $\mathrm{ARI}=0$ & $A R I=1$ & $\mathrm{ARI}=2$ & $A R I=3$ \\
\hline Group I Halogen No cycles & 15 & $3(20 \%)$ & $10(66.7 \%)$ & $1(6.7 \%)$ & $1(6.7 \%)$ \\
\hline Group II Halogen 500 cycles & 15 & $3(20 \%)$ & $11(73.3 \%)$ & $1(6.7 \%)$ & $0(0 \%)$ \\
\hline Group III Halogen 10,000 cycles & 15 & $5(33.3 \%)$ & $9(60 \%)$ & $1(6.7 \%)$ & $0(0 \%)$ \\
\hline Group IV Plasma Arc No cycles & 15 & $6(40 \%)$ & $8(53.3 \%)$ & $1(6.7 \%)$ & $0(0 \%)$ \\
\hline Group V Plasma Arc 500 cycles & 15 & $2(13.3 \%)$ & 12 (80\%) & $1(6.7 \%)$ & $0(0 \%)$ \\
\hline Group VI Plasma Arc 10,000 cycles & 15 & $1(6.7 \%)$ & $14(93.3 \%)$ & $0(0 \%)$ & $0(0 \%)$ \\
\hline
\end{tabular}


cling include the differences in the coefficient of thermal expansion among the adhesive, metal bracket, and enamel. The cyclical stress of thermocycling at two different temperature extremes could also cause some weakened areas within the bond to grow progressively in size. ${ }^{16}$ Another theory involves the absorption or solubility of the composite after thermocycling. This procedure causes hygroscopic expansion as well as chemical degradation of the materials. It has been shown that composites that were thermocycled absorb more water than those that were not thermocycled. ${ }^{16,17}$

Our findings showed that thermocycling affected the SBS of both light sources. We want to point out the significant decline of the PAC group. In addition, it is known that rapid curing may not allow sufficient time for the pre-gel phase of the material to absorb polymerization/contraction stresses. ${ }^{18}$ This partial polymerization can increase the water absorption, which can affect the longevity of the restoration. ${ }^{19,20} \mathrm{An}$ adhesive layer that is very thin in the orthodontic brackets may be more affected under the thermal cycle of the PAC than the halogen-light source. Therefore, the SBS values may show significant reduction in the PAC group.

The 10,000 thermocycles correspond to approximately one year under humidity and temperature changes. ${ }^{21}$ However, the response to the 10,000 thermal cycle procedure was different for both light sources. The reduction in SBS was decelerated by 2.5 percent in the PAC-light group. Under the same procedure, the halogenlight group showed a greater reduction of about 26.4 percent. The PAC group showed a significant reduction under 10,000 thermocycles, as did the halogen group. Interestingly, there was a regular decrease in the SBS values in the halogen group, while no decrease was observed in the PAC under 10,000 cycles. The negative effect of the high intensity of the PAC may be normalized or adapted after a particular amount of time. Therefore, the response of the PAC to the humidity and temperature changes were similar to that of the halogen light at the end of 10,000 cycles.

Similarly, Manzo et al ${ }^{22}$ investigated bracket failure in patients in halogen and PAC groups. The follow-up occurred for approximately 406 days, and no statistical difference was observed.

The ARI was developed to quantify the amount of adhesive that remains on the bracket after a bracket base is debonded. The ARI scores did not show any statistical differences between the light sources. Before thermocycling, failure of the bracket was observed at enamel-adhesive (score 1). After thermocycling, the predominant scores did not change in both groups. However, it was observed that the halogen group shifted toward ARI scores of 0 with the increasing the number of cycles. Increasing the number of thermocycles caused less adhesive to remain on the tooth. However, the bracket failure of the PAC group shifted to scores of 1. However, mainly, the thermocycling procedures did not affect the bracket failure in either group. The failure of this type ladhesivebracket) may be advantageous for removing residual adhesives from the teeth. On the other hand, the enamel surface can be damaged when failure occurs in this mode.

With respect to the clinical aspect of this study, the two methods have been successful under the thermocycle procedures. However, the PAC seems to be more beneficial than the halogen-light cure; approximately 5 minutes could be saved for the complete upper and lower arch. This chair time saved reduces the risk of saliva contamination and an increase in the pulpal temperature, as well as reducing further incidences of bond failure. The disadvantage of the PAC is that its cost is approximately six times higher than that of a halogen light.

\section{CONCLUSIONS}

- Both light sources showed favorable SBS performance without the thermocycle procedure.

- Thermocycling significantly decreased the SBS values of both groups.

- After 10,000 thermocycle procedures, the response of both the light sources, i.e. SBS values, was similar.

- The ARI scores under the thermocycle procedures showed no differences between the two light sources.

\section{REFERENCES}

1. Thind BS, Stirrups DR, Charles HL. A comparison of tungsten-quartz-halogen, plasma arc and light-emitting diode light sources for the polymerization of an orthodontic adhesive. Eur J Orthod 2006;28:78-82. 
2. Tavas A, Watts DC. Bonding of orthodontic brackets by transillumination of a light-activated composite: an in vitro study. Br J Orthod 1979;6:207- 208.

3. Sfondrini MF, Cacciafesta V, Scribante A, Klersy C. Plasma arc versus halogen light curing of orthodontic brackets: $A$ 12 month clinical study of bond failures. Am J Orthod Dentofacial Orthop 2004;125:342-347.

4. Dunn WJ, Bush AC. A comparison of polymerization by light-emitting diode and halogen-based light-curing units. J Am Dent Assoc 2002;133: 335-341.

5. Mills RW, Jandt KD. Blue LEDs for curing polymer based dental filling materials. Laser Electro-optics. Electron Devices Society Newsletter 1998;12:9-10.

6. Krishnaswamy NR, Sunitha C. Light-emitting diode vs halogen light curing of orthodontic brackets: A 15- month clinical study of bond failures. Am J Orthod Dentofacial Orthop 2007;132: 518-523.

7. Oyama N, Komori A, Nakahara R. Evaluation of light curing used for polymerization of orthodontic bonding agents. Angle Orthod 2004;74:810-815.

8. Pettemerides AP, Ireland AJ, Sherriff M. An ex vivo investigation into the use of a plasma arc lamp when using a visible light-cured composite and a resin-modified glass poly (alkeonate) cement in orthodontic bonding. J Orthod 2001;28: 237-244.

9. Kiremitci A, Yalcin F, Gokalp S. Bonding to enamel and dentin using self-etching adhesive systems. Quintessence Int 2004;35:367-370.

10. Artun J, Bergland S. Clinical trials with crystal growth conditioning as an alternative to acid-etch enamel pretreatment. Am J Orthod 1984:85:333-340.

11. Cehreli ZC, Kecik D, Kocadereli I. Effect of self-etching primer and adhesive formulations on the shear bond strenght of orthodontic brackets. Am J Orthod Dentofacial Orthop 2005;127: 573-579.

12. ISO Technical Report 11405. Dental materials-guidance on testing of adhesion to tooth structure. Geneva: International Organisation for Standardization 1994.

13. Reynolds IR. A review of direct orthodontic bonding. $\mathrm{Br} J$ Orthod 1975;2:171-178.

14. Oesterle LJ, Newmann SM, Shellhart WC Rapid curing of bonding composite with xenon plasma arc light. Am J Orthod Dentofacial Orthop 2001;119:610-616.

15. Daub J, Berzins DW, Linn JB, Bradley TG. Bond strength of direct and indirect bonded brackets after thermocycling. Angle Orthod 2006;76:295-300.

16. Arıcı S, Arıcı N. Effects of thermocycling on the bond strength of a resin- modified glas ionomer cement: An in vitro comparative study. Angle Orthod 2003;73:692-696.
17. Yap AU, Wee KE. Effects of cyclic temperature changes on water sorption and solubility of composite restoratives. Oper Dent 2002;27:147-153.

18. Sakaguchi RL, Berge HX. Reduced light energy density decrease post-gel contraction while maintaining degree of conversion in composites. J Dent 1998;26: 695-700.

19. Rueggeberg FA, Caughman WF. The influence of light exposure on polymerization of dual-cure resin cements. Oper Dent 1993;18:48-55.

20. Mehl A, Hickel R, Kunzelmann KH. Physical properties and gap formation of light-cured composites with and withoutsoft-start polymerization'. J Dent 1997;25:321-330.

21. Gale MS, Darvell BW. Thermal cycling procedures for laboratory testing of dental restorations. J Dent 1999;27:89-99.

22. Manzo B, Liistro G, De Clerck H. Clinical trial comparing plasma arc and conventional halogen curing lights for orthodontic bonding. Am J Orthod Dentofacial Orthop 2004;125:30-35. 